\title{
Endocrinolog $\mathrm{y}$ \\ The Bulletin of the Association for the Study of \\ Internal Secretions
}

. September, 1921

\begin{abstract}
THE SIGNIFICANCE OF THE INTERNAL SECRETIONS
\end{abstract} IN DISTURBANCES OF METABOLISM AND DIGESTION

Report given to the Homburg Meeting for Diseases of Metabolism and Digestion, September 26, 1920.

PROF. DR. ARTHUR BIEDL

PraG

Netabolism, this continuous exchange of material and energy in the organism takes its origin in chemical processes, and must, therefore, naturally represent the chief field of activity of chemical regulations. One can well say that in all departments of biology the tenets of the internal secretions have extended themselves during the last thirty years to an unexpected expansion and depth. They have taught us that the chemical messengers of all tissues, but before all the endocrine glands, are concerned with development, formation, and growth in its progressive and regressive phases, in the somatic and psychic constitution of the individual, and that the degree of activity of all the tissues, organs and systems is also more or less influenced. It has also taught us that all hormonal correlations in spite of their phenomenal multiplicity are in the last analysis a matter of a direct or indirect alteration of the biochemistry, in its widest sense, the metabolism. In order to represent correctly the relation of the internal secretions to metabolism, it would be actually necessary to consider the whole realm of endocrinology in extenso. We will, therefore, confine ourselves to the discussion of the diagnostic significance of the internal secretions in disturbances of metabolism and the digestive organs. Thus the study can be 
taken up on the basis of the relations that have so far been ascertained between endocrine organ activity and metabolism, and calling attention to those phases where in individual cases the metabolic participation, particularly its first preparatory phase, digestion, in healthy and diseased conditions can be charged up to the endocrine glands. It is my intention to treat not only of the role of the science of the internal secretions in the diagnosis of disorders of metabolism and digestion, but much more will the questions be answered regarding the influence of the endocrine glands individually and as a whole on the magnitude and course of metabolism. We will also consider their influence on the variations in disease and the kind, intensity and course of these variations, and finally that which is most important for the physician how and what kind of a point of attachment can be gained for a workable therapeutic treatment of the diseases from the extensive information at hand.

From the experimental and clinical analytical studies on the physiological role of the endocrine glands, the general conclusion presents itself before all others that the range and course of metabolism, in its total exchange with its two parts, basal metabolism and production efficiency, just as in its individual components ; protein, fat, carbohydrate, mineral and water exchange, to a much greater degree than has previously been recognized, is directed and regulated by the endocrine glands. From this it follows further that in our critical examination of metabolism, we must utilize all those points of attachment more than we have before which will put us in a position to obtain more intimate data concerning the individual endocrine glands, or more exactly in regard to the endocrine formula of the individual. The way is known to us. In countless individual cases the important influence on morphogenesis during embryonic and post-embryonic development of the glands of internal secretion is thoroughly established, and has been proven in growth and adult life. It shows itself in the constitution of the individual, the outer appearance of the bodily constitution, the habitus, has with its related single components served as a measure of the critical judgment of physicians from earliest times. Even today we can only judge of the constitution when we can study the unit components of the organic makeup by measuring, weighing and other comparative evaluations. They are as follows: the body weight; Downloaded frof 
the body length as an index of the growth of the long bones; the breadth of the chest as an indication of the skeletal development of the trunk; the mass and tonus of the musculature; the firmness of the fat deposits; the condition of the skin, and its appendices, the hair and nails. I may indeed suppose that the facts concerning the role of the growth-influencing glands on the habitus and constitution are well known. It accordingly seems superfluous to me to analyze more closely those variations which can be attributed to abnormal conditions, and particularly to the quantitatively varying function of one or more of the glands on the proportionality of the body structure, on the integument, and the sexual landmarks, on the irritability and activity of the neuro-muscular apparatus and the vegetative organs, and on the temperament and the psychic idiosyncrasy of the individual. Having gathered together the individual findings we can say: when we have grasped the bodily organization of an individual in toto and when we have correctly analyzed its single component factors on the basis of the previous and increasingly extensive information-we are still unfortunately very far from an exact and measured constitution analysis, the first weary steps of which have now been taken by Hammar in Upsala-then the internal secretory constellation, the endocrine formula is so far made clear that we now have obtained a new standpoint for the critical examination of the metabolism of the individual. We will first of all oppose the so-called standard values for the magnitude of the energy consumption and for the nature of the dietary needs and consumption, which have been obtained from many studies, with the acknowledgment that these can scarcely help us in our practical experience. For it is clear that actually different standard values must serve for normal function, for the over-function and the under-function of the individual endocrine glands. Hence the terminology, hypo- and hyper-thyroidism, hypo- and hyper-pituitarism, hypo- and hyper-genitalism are in reality only. schematic, separating concisely conditions which are not determinable quantitatively and which cannot be separated from each other by sharp boundaries. Therefore, we are com. pelled to prepare individual standard values. The average values extremely important for statistics and political economy have lost their significance in the clinic. In the individual we see farreaching differences not only in the amount but also in the kind 
of the food ingested, we find differences in the nutritive condition, in which no parallelism can be found between the amount of food intake and the body weight, used as an index of the condition of nutrition; in fact quite often a marked discrepancy exists. I will call attention to a well known example, a problem which confronts us in the light of the theory of internal secretions. That is obesity. It is incontestable that adiposity can only arise when the food ingestion exceeds the energy needs of the body for a prolonged period. In other words, no other form is conceivable than the adiposity of overfeeding. The experience, that there are individuals who for the most part come from families with hereditary adiposity without detectable overfeeding and without marked diminution of muscular activity, who become fat and who even remain fat with an adequate restriction of food intake leads to the origin of the term "constitutional adiposity." It is obvious that countless studies have been undertaken to show that the cause of this condition is a metabolic anomaly, and to discover whether it is due primarily to a lessened energy output and further whether the condition arises from a lower catabolism or from a lowered efficiency of production, be it through a diminution of the specific dynamic: action of the foodstuffs, or be it because of a greater utilization of the energy derived from the food during work. A closer exposition of these studies is superfluous. We know that the sum total of the previous researches have afforded no decisive proof either for a lowering of the catabolism, or for a lowering of the decomposition energy of protoplasm in obesity. The lower values, particularly those found in the prolonged experiments of v. Bergmann could indeed, in this sense, be quoted. I might recall to your minds the much more important demonstrations of the observations, first made by v. Noorden, and since then by other clinicians, that in many cases of obesity there is no loss of body weight even when extraordinarily small amounts of calories are ingested.

The principal objection which we raise against the respiration studies is that they cannot answer the questions that have been put to them. For apart from the fact that the short-period methods are complicated by errors, which are large enough to explain the genesis of adiposity by a diminution of catabolism, the very marked variations in basal metabolism shown in v. BergDownloaded from https://academic.oup.com/endo/article-abstract/5/5/523/2771550 
mann's tables afford no valid conclusions if the figures obtained are compared with the average values. We have no right to draw conclusions from this comparison. Neither the average energy need, while resting, nor the average production efficiency during muscular work can serve as a basis for the comparison. We must a priori expect far-reaching differences in different individuals if we but recognize the different original size and the still more marked variability of the function magnitude of the endocrine organs. This contention is confirmed by the results of the metabolism experiments. The values obtained from different individuals, even when reckoned on the basis of body weight or body surface, vary within such wide boundaries that one indeed cannot use the average values obtained in the fasting condition for the individual for the calculations. Let us pass along. The basal metabolism as a measure for the work, which in the resting, fasting organism is represented by the vegetative organs and the maintenance of the normal muscle tone, must change with a change in the operative factors. The endocrine glands stand in the first rank as directive agents of these phenomena. In a similar manner the influence of the endocrine glands on the efficiency of production is also availing in that appetite, nutrition and impulses to movements are dependent upon them in part as are the strength and duration of muscular activity. Now the small unmeasurable muscular movements of the daily life whose number and magnitude are dependent upon the agility and the temperament of the individual, or more acurately his endocine glands, yield in their totality undoubtedly an energy consumption that is not to be discounted. That production efficiency differs strongly at different ages according to the magnitude of the muscular activity is self-evident. That metabolism differs at different ages is a matter of record. But too little attention has been paid to the fact that primary differential endogenous factors are determinative for the differences in muscle tonus and for the different stimuli to muscular activity as is commonly demonstrated by the impetuosity of youth, the restraint of the movements of the adult, and in the increased comfort-seeking of the middle-aged and in the high degree of rest needed by the aged.

Although the individual energy need is defined in its two components by endogenous factors, and since these show indi- 
vidual variations of the widest degree, so we are no longer able to make constitutional adiposity dependent on the proof of a slowing of nutrition but must in consequence consider every adiposity as constitutional as endogenously conditioned. We can, of course, formulate the conception in another way and say: every adiposity is a dietary adiposity which does not arise absolutely through over-feeding, but only through an individual call for excessive food intake. Of prime importance are the endogenous factors of the endocrine glands.

However obvious this point of view may appear, or however little it contains of what is new or surprising, it is a matter of great interest that when individual cases are subjected to other methods of investigation we thereupon acquire definite directions for an efficient therapy. We are no longer concerned in the analysis of the respiratory gases with the comparison with standard values, but turn to the more exact method of experimentation. After a careful weighing of the points of control given by the clinical signs and after the establishment of the individual standards which also undergo variations, experimentally active amounts of one or the other or several glands of internal secretion are administered and whether or not a change in the catabolism can readily be obtained is determined. A constant detectable marked increase in the metabolism which is caused by a certain hormone or a definite hormone combination and by this only proves indeed that the individual in question has a lower exchange as a personal standard than is stipulated in hormonal equilibrium. At the same time we have also ascertained the cause of the equilibrium disturbance.

The practical clinical continuation of this method of research shapes itself up rather simply; its results are none the less striking. We use the body weight as the measure of metabolism, and can easily find a normal diet on which the body remains constant. Now the administration in small quantity of a definite hormone, one day's administration is sufficient, occasions a distinct alteration of the body weight safely over that imposed by the probable error of the determination: Ceteris paribus such an alteration is not to be expected from any other hormone or any other hormone combination. We have determined in this manner the endogenous factors of the disordered metabolism, and the prompt, often overwhelming, effect of the organo-therapy Downloaded from https://academic.oup.com/endo/article-abstract/5/5/523/2771550 
which has been carried on strengthens the justness of our conclusion. In many cases no difficulties are encountered in foreseeing with great probability from the clinical character of the adiposity the pathogenetic determinative endocrine gland. I can well omit the description of the differential landmarks of the thyreogenous, hypophyseal, genital and other types of endogenous adiposity, but I must call attention to the fact that in the more exact clinical analysis one runs across a not inconsiderable number of combination types. It is important that the hormone study and consequent hormone therapy should make genetically clear not only the simple but also the combination cases, and thus make possible an effective therapy. I can cite many cases from my experience in which, on the basis of the clinically determined endocrine formula in hormone research, the disturbing endocrine glands were recognized as it were at the first attempt at hormone investigation, and the further study with other hormones, by their negative results, confirmed the exclusive significance of the single organ. For example, when a case of adiposity that had already been clinically recognized as certainly of thyreogenous origin was treated with a tenth of a gram of desiccated thyroid substance three times a day, while ingesting a standard diet and while at a constant weight, the weight dropped half a kilo in 24 hours, and in six days 2.5 kilos were lost. The same case remained at a constant weight when any other hormone was given even in large amounts, and when thyroid preparation was again given the same prompt action was obtained. On the other hand, a case of obstinate obesity which occurred during the lactation period did not react at all to thyroid administration, but responded promptly to ovarian tablets. For the combination types one must first ascertain the suitable mixture of glandular preparations to be used: the last year I have used in my researches an American preparation in which several endocrine glands are contained in variable mixtures.

Of particular interest are just those cases in which the clinical signs are indeterminate, even under certain conditions misleading. An example of this is a nineteen is ar-old young man with heavy fat deposits, particularly on the breast, in the mammae, the lack of any trace of hair on the face, on the trunk lower abdomen, on the hips and nates. The markedly developed 
and on the thighs, the lack of axillary hair and the especially sparse pubic hair taken together with infantile external genitals and hypoplastic testicles, with a plainly effeminate expression, suggest an outspoken Fröhlich type. No definite indication could be found either of an hypophyseal disturbance or of an intracranial tumor. We were confined to the hypothesis of a primary hypogenitalism. But neither testicular nor ovarian substance had the slightest ability to cause an increase in metabolism. Before testing out other hormone substances, the short stature or rather the markedly short body length of $156 \mathrm{~cm}$. accompanied by only a few suggestive signs of hypothyroidism, such as the hair and nails, led to a test with thyroid substance, which was strongly positive. The administered thyroid substance effected promptly the desired decrease in body weight from $2 \frac{1}{2}$ to 3 kilos a week. The thyroid therapy was reduced to half the quantity after the diminution in weight had reached 18 kilos. After five months' treatment there was presented to me the now 191/2-year-old youth as a slim $160 \mathrm{~cm}$. tall man with a perceptible growth of beard, so that he must shave, and with the distinct astonishing habitus of a grown man. On the anterior side of the trunk the abnormal fat deposit was lacking, which now was only to be found on the buttocks. Axillary hair had grown in, profuse pubendal hair of the male type reaching to the navel, hairy thighs, and almost completely developed external genitalia corresponding to the age and with correspondingly large testicles. The voice and psyche were completely male; the total loss of weight was 26 kilos.

This case impressively teaches us the correlative tying up of metabolism and the whole body organization with a dependence on the endocrine apparatus, but it shows us, also, the prevalence of a link of the endocrine chain, which indeed occurs in many cases, which, however, is not always schematically demonstrated, and must first be proven by experimental tests. The dominating significance of any one endocrine gland can scarcely be exhibited in the combination types of treatment. One should be particularly warned against this because it is a tendency of modern times "to cure all from one point." If we consider old age as a problem of metabolism-and this opinion undoubtedly everyone will agree with-so then one will recognize my right at this opportunity to say some words concerning old age and revert Downloaded fich 
to the recent question of averting senescence, or rather rejuvenation, which from the time of Brown-Sèquard has been of great dramatic interest. The "father of the internal secretions" placed the gonads as the axis of the problem of old age, and it is perhaps not superfluous to call to mind that the communications of this old man on his studies with the "liquide testiculaire," which he consistently administered to himself, had such scintillating confirmatory results, and similarly the evident attention which was directed to him for thirty years, which still survives to us today.

But if today one should make the gonads answerable for senesence, or more exactly those tissue elements designated by Steinach as the pubertal glands, and if one should abolish completely or avert the whole complex of old age or even one portion of the phenomenon by the reanimation of these cells, then one would overlook the important development which the teaching of Brown-Sèquard has accomplished and the results afforded by the earlier workers would be forgotten, namely, the recognition of the significance and the effect of the other endocrine glands and their co-operation.

Just as all bodily conditions and their fundamental chemical reactions are under the influence of the entire hormone apparatus, so is old age. This condition is regulated and directed in the degree and course of its development, not only by the testicles or ovaries, particularly the interstitial cells, but just as well by the thyroid and thymus glands, by the hypophysis and the pineal, by the inter-renal and the adrenal system, and perhaps even by other organs and tissues, in short by the whole endocrine apparatus, by its single parts, but before all by the harmonious cooperation of these parts. It seems to me quite superfluous to direct attention to the fact that in cases of premature old age on the ground of certain indications, that are perhaps striking only to him who recognizes them, that in the one case the thyroid, in the other the hypophysis or the adrenals, and even other endocrine glands can be considered as the pathogenetic centers. At times the opinion made at the time of observation is verified at autopsy. When tissue transplantation or hormone administration is used as a method of substitution therapy, rejuvenation effects can doubtless be obtained. Or how else can one interpret the fact that there is produced a surprising alteration of the entire individual by feeding thyroid to a cretin, to choose a 
familiar example, whose appearance and vegetative and somatic functions are those of an old person.

In a case of ateleiosis (defective development) - the tenyear-old girl had the appearance, the metabolic, the somatic and psychic condition of an old woman-by a combined hypophyseal ovarian treatment I unexpectedly obtained the result that, together with a gradual transformation of the skin, the hair, the teeth and the nails, the increased muscle tonus and the fat pads of adolescence, there began a growth in body length and after about a six months a real child stood before me, who, however, still carried several characteristics of her earlier condition.

I will not be prolix, it is not my intention and indeed here is not the place to speak critically of Steinach's work. From my point of view, if the physiological role of a specific endocrine gland can be determined by safe proof and exact experimentation, so that we may be able to actually seize hold of the organic machinery on the basis of such determination, such a finding must be greeted with pleasure. Whether and how far Steinach's studies meet this postulate will be considered by me in another place. Here I can only touch on the fact that old age and youth are not entirely dependent on the pubertal glands, but that they bring up questions of endocrine harmony, a solution for which can only be obtained by bringing into use all methods of research on the internal secretions.

After this deviation we will now return to the main theme and, however needlessly, remark that all those viewpoints which are valid for the plus deviation of metabolism (adiposity) are also valid for the minus deviation (emaciation). As previously mentioned, this is endogenous and rests largely on an increased activity of glands that accelerate metabolism. The opposite case, the suppression or the decreased action of retardent endocrine glands, practically plays a much smaller rôle. Unfortunately, we do not possess any similarly acting substitution-therapy or easily applicable methods suitable for causing a retardation of an hormone over-function when it exists. The Roentgen and radium-rays act almost only in a destructive manner-definitely in their effects as function diminishers; perhaps it is only a question of technique to evolve methods for the attainment of temporary effects. By the attainment of this preliminary condition we can effectively attack idiopathic, constitutional and even by OGI School of Science and Engineering at OHSU user 
other forms of emaciation, such as today is done in the diet cures. I will briefly relate a striking case in which organotherapy was effective. Hofstätter, as is well known, used pineal extracts with good result in combatting pathologically increased libido sexualis. And Pilcz, on his recommendation, tried the same treatment in a case of dementia precox with favorable results. In a second case of this type Hofstätter's proposal of pineal medication was carried out and I had the opportunity of observing the case myself. Direct points of attachment for a diagnosis of hypergenitalism or a hyperactivity in a sexual sphere were lacking, the only marked symptom was the high degree of emaciation of the patient in spite of his abnormally great ingestion of food. The constant activity and increased muscle tonus made the increased caloric consumption understandable. The oral administration of a commercial preparation of alleged pineal substance was completely ineffective. The subcutaneous administration of a laboratory pineal preparation produced even after a short time a reduction of the activity and a noticeable increase of the body weight-the total increase amounting to 16 kilos-notwithstanding a decreased food intake. On the basis of these results I would use organo-therapy with pineal substance in certain cases of constitutional emaciation, but unfortunately we are today contending with the difficulty of procuring a dependable material. It might be said in passing that this applies equally well to all other spheres of endocrine preparations. Standard tests for such preparations and their physiological assay are urgently needed.

If we now direct our attention to the single factors of metabolism instead of the general, it is clear that these are under the control of the glands of internal secretion, that the need and consumption of the single foodstuffs and the manner of their decomposition in the organism are influenced by the endocrine glands as individuals and as a whole. The functional state of the individual endocrine gland must be thought of when considering the magnitude of the protein minimum, which, according to earlier studies, is likewise a matter of individuality, just as are the external factors which previously almost exclusively have been alone considered. That many of the latter are ultimately dependent on the temperament of the endocrine glands sounds at present like a snap judgment. It is entirely unneces- 
sary to make further remarks upon the influence of every single gland on protein, fat, carbohydrate and salt metabolism. The diseased conditions of the endocrine glands as such and their consequence on metabolism will not be spoken of further here. The place of the endocrine glands in the pathogenesis of any individual metabolic disease demands a report by itself.

A brief-consideration of the significance of the internal secretions in digestion is now in order. In diagnosis the influence of the new ideas are especially noticeable in two directions: first, the great field of the size, form, anomalies of position and changed conditions are no longer, as formerly, only handled exclusively as descriptive anatomy, or are the best cases considered embryologically and teratologically, but they are rather more looked upon as dovetailing into the total body makeup as partial constitutional components in a casual genetic analysis, in which proven morphogenic influence of the hormone glands serves as a basis. Secondly, the nervous system is not made exclusively responsible from the functional point of view for their physiological variations and pathological alterations, but the fact is being brought into increasing prominence that the chemical messengers of the endocrine glands in their activity, direct or indirect, play a regulating part, through mediation of the sympathetic and para-sympathetic nervous system, and that a hormone action deviating from the normal can play a disturbing rôle in functional disturbances.

This progress illustrates very prettily the angles of view from which we today consider those helpers of the digestive. apparatus, the teeth. We know today that the regulation and properties of many endocrine glands are responsible for the observed anomalies of teeth formation and structure. That by all means rare persistence of the milk teeth and the frequent single milk teeth can no longer be considered as curios, but as expressions of a partial infantilism, for whose origin the branchiogenous organs, the thyroid and hypophysis, as was to be expected, come into play. The enamel defects of the incisors are the long expected document of a parathyroid insufficiency occurring in the earliest years of childhood, and as such point to the utilization of this organ. From the studies of Kranz, Josefson, and others, we have obtained information as to the influence which

the thyroid exerts on the growth of the maxilla and dentition, Downloaded from hteps://academic.oup.com/endo/article-abstract/5/5/523/277155 
and on the spacing and peculiarities of the teeth. We must consider from an altered point of view the question of dental caries and alveolar pyorrhea, since the experimental results are always forcibly impressive with respect to endocrine and metabolic factors. The morphological anomalies in the digestive tract itself have assumed a new aspect, since the various forms of stenoses, dilatations and diverticular formation in the oesophagus, the place and structural anomalies of the stomach and intestine are none other than infantilisms and can be considered as signs of further developed constitutional variation conditioned by the glands of internal secretion, occurring in fetal life and during the growth period. The necessary individual observations and experiences in this field are indeed still few. To a certain extent these apparent anatomical alterations and the functional disturbances following them fall in the broad terrain of the muscle tomus of the vegetative organs. It would naturally lead very far if one were to treat the tonus problem in its entirety. Only one thing is to be noted, and that is that even if today the nature of touus is by no means sufficiently clear and even if the question of the part of the sympathetic, parasympathetic and central nervous systems figure in the discussion of tonus, the dependency of tonus on and its influencing by the endocrine glands is undoubted and is directly proven in many cases. Diagnostically and therapeutically we can deduce important conclusions, under certain circumstances, from this state of affairs. I call to mind - to give one example only-the action of the hormone of the thyroid or the pars intermedia of the hypophysis on the tonus of smooth musculature and to the generally known striking consequences of thyroid medication in certain forms of habitual constipation apparently of thyreogenous origin. Many times we have to do with the common effect of several endocrine glands in pathological changes and diminution of tone. Here pluriglandular therapy comes into its own on the basis of the clinical point of attack.

The principles for the secretion disturbances of the digestive tract are the same as those for the anomalies of tonus and motility. I can, therefore, consider them briefly. The progress of the studies of constitutional makeup are putting us in a position, to an increasing degree, to appraise the magnitude of the endocrine components in the origin and shaping up of the ground, on 
which a pathological impetus can give rise not only to abortive functions in increasing intensity, but also to an anatomical destruction.

It is only necessary here to call attention to the relation of the adrenal system to the origin of gastric ulcer, which indeed is not yet definitely proven, and particularly the most recent study reported from Russia in which the important rôle of the different endocrine organs for the origin, growth and the retrogression of malignant tumors in animals is discussed.

That the magnitude of the functions of the secretory apparatus of the digestive tract is intimately related to the endocrine system needs no particular exposition, if we call to mind the fundamental researches that have been made in just this field. Many digestive organs, such as the liver and the pancreas, produce simultaneously an external and internal secretion, they are of the greatest significance for the preparatory phase of digestion and also for total metabolism. They are, however, at the same time links in the chain of the endocrine glands and are connected with all the other links in a most intimate interchange of effectiveness. It would be very enticing to expand more extensively on this very attractive theme.

In the conclusion of this discussion may I especially point out that I have been able to handle this extensive sphere only in programmatic brevity and, as it were, as a sketch planned to. touch the high points in a penetrating presentation. I may welf hope, however, that I have perhaps succeeded in directing notice to a province of biological study that still needs many active workers, and is indeed worthy of them. 